\title{
Importance socioéconomique de Elaeis guineensis Jacq. (Palmier à huile) en Basse-Casamance (SENEGAL)
}

\author{
Boubacar Camara \\ (Université Assane SECK de Ziguinchor/Senegal) \\ UFR Sciences et Technologies, Laboratoire d'Agroforesterie et d'Ecologie) \\ Institut Sénégalais de Recherches Agricoles \\ (Centre National de Recherches Forestières Maristes/Dakar Sénégal) \\ Boubacar Sagna \\ (Université Assane SECK de Ziguinchor/Senegal) \\ UFR Sciences et Technologies, (Aménagement et Gestion Durable des \\ Ecosystèmes Forestiers et Agroforestiers) \\ Université Assane Seck de Ziguinchor/Sénégal \\ Daouda Ngom, (Professeur, PhD Agro-Ecologue) \\ (Université Assane SECK de Ziguinchor/Senegal) \\ UFR Sciences et Technologies, (Laboratoire d'Agroforesterie et d'Ecologie) \\ Mamadou Niokane \\ (Ingerieur Agronome, Agro-economiste) \\ Groupe de Recherche et de Réalisations pour le Développement \\ Rural/Cellule de Ziguinchor/Sénégal \\ Zoe Daba Gomis \\ (Université Assane SECK de Ziguinchor/Senegal) \\ UFR Sciences et Technologies, (Aménagement et Gestion Durable des \\ Ecosystèmes Forestiers et Agroforestiers) \\ Université Assane Seck de Ziguinchor/Sénégal
}

doi: 10.19044/esj.2017.v13n12p214 URL:http://dx.doi.org/10.19044/esj.2017.v13n12p214

\begin{abstract}
Lower Casamance harbors enormous economic potentialities due to the importance of its forest resources. Among the forest species of the area, Elaeis guineensis is one of the most exploited for its many services and products. Despite its importance, the oil palm tree is experiencing real threats linked to a combination of natural and anthropogenic factors. The overall objective of this study is to contribute and evaluate the goods and services provided by the agroforestry parks in Lower Casamance which will lead to an alert to the whole community about the threats to these secular systems.
\end{abstract}


For that purpose, an individual questionnaire was administered to a sample of 146 invidious (33 in Carounate, 57 in Kabiline and 56 in Kaguite) according to their business sector in the palm plantation in each of the sites. Corresponding to $5 \%$ poll rate. It should be noted that most of the surveyed population acquires their product through harvesting (72\% in Carounate, $70.97 \%$ in Kabiline and 70.21 in Kaguite). All parts of the oil palm are exploited by the local population for various purposes. Thus the most important frequencies are the use in construction $(100 \%)$, the production of palm oil on average $(94.87 \%$ and the making of brooms on average $(92.97 \%)$. The sale of the palm products is a source of unshakeable revenue that can provide up to $865000 \mathrm{~F}$ cfa for palm oil, 675000Fcfa for palm wine and $280000 \mathrm{~F}$ cfa for brooms. This is why, according to $90.91 \%$ of the respondents in Carounate, $37.41 \%$ in Kabiline and $45.71 \%$, the exploitation of the oil palm occupies the second place, after agriculture, in their activities. It is therefore all the interest to permanently preserve these secular systems.

Keywords: Importance, socioeconomic, Elaeis guineensis Jacq.,Lower Casamance SENEGAL.

\section{Résumé}

La Casamance recèle d'énormes potentialités économiques de par l'importance de ses ressources forestières. Parmi les espèces forestières de la zone, Elaeis guineensis fait partie des plus exploitées pour ses nombreux services et produits. Malgré son importance, le palmier à huile connait de réelles menaces liées à une combinaison de facteurs naturels et anthropiques. L'objectif général de cette étude est de contribuer à l'évaluation des biens et services fournis par les parcs agroforestiers en Basse Casamance. Pour cela, un questionnaire individuel a été administré à un échantillon de 146 personnes (33 à Carounate, 57 à Kabiline et 56 à Kaguite) selon leur secteur d'activité dans la palmeraie dans chacun des sites correspondant à un taux de sondage de 5\%. Il convient de retenir que la majorité de la population enquêtée acquiert leurs produits à travers la cueillette $(72 \%$ à Carounate, $70,97 \%$ à Kabiline et 70,21 à Kaguite). Toutes les parties du palmier à huile sont exploitées par la population locale pour divers besoins. Ainsi les fréquences les plus importantes sont l'usage dans la construction (100\%), la production d'huile de palme en moyenne $(94,87 \%$ et la confection de balais en moyenne $(92,97 \%)$. La vente des produits du palmier est une source de revenus non négligeable pouvant fournir jusqu'à $865000 \mathrm{~F}$ cfa pour l'huile de palme, $675000 \mathrm{Fcfa}$ pour le vin de palme et $280000 \mathrm{~F}$ cfa pour les balais par an. C'est pourquoi, selon $90,91 \%$ des enquêtés à Carounate, $37,41 \%$ à Kabiline et 45,71\%, l'exploitation du palmier à huile occupe la deuxième 
place, après l'agriculture, dans leurs activités. C'est donc tout l'intêret de conserver durablement ces systémes séculaires.

Mots-clés : Importance, socioéconomique, Elaeis guineensis Jacq., BasseCasamance SENEGAL

\section{Introduction}

La Casamance, région sud du Sénégal, recèle d'énormes potentialités économiques de par son réseau hydrographique dense, ses terres relativement fertiles, et la présence de ressources agricoles (céréales, arboriculture, horticulture, etc.), de ressources halieutiques (poissons, huitres, coquillages, crevettes etc.) et de ressources forestières (bois, fruits, miel, plantes médicinales...). Ces dernières occupent une place importante dans la subsistance des populations. A cet effet, en dehors de l'agriculture, de l'élevage ou de la pêche, la cueillette des produits forestiers non ligneux constitue pour les communautés rurales une source importante de revenus, d'aliments et de médicaments (Ezebilo et Mattsson, 2010). Parmi les espèces forestières de la Basse Casamance, Elaeis guineensis fait partie des plus exploitées pour ses nombreux services et produits tels que l'huile de palme, l'huile de palmiste, le vin, les matériaux de construction, les médicaments, le savon, les engrais etc. (Carrere, 2010).

Cependant, les parcs agroforestiers à Elaeis guineensis subissent de réelles menaces liées à une combinaison de facteurs naturels et anthropiques. L'objectif général de cette étude est de contribuer à l'évaluation des biens et services fournis par les parcs agroforestiers en Basse Casamance. Les résultats constitueront des indicateurs pour alerter toute la communauté sur les menaces qui pèsent sur ces systèmes séculaires.

\section{MATERIEL ET METHODES Choix des sites}

La Basse Casamance correspond à la région administrative de Ziguinchor. Elle se situe dans la partie méridionale du Sénégal. Elle s'étend sur une superficie de $7300 \mathrm{~km}^{2}$. Elle est limitée au Nord par la Gambie, au Sud par la Guinée Bissau, à l'Ouest par l'Océan Atlantique et à l'Est par la rivière Koulountou (région de Sédhiou). Elle a été érigée en région administrative en juillet 1984 et englobe ainsi les départements de Ziguinchor, de Bignona et de Oussouye (Niane, 1984,). Ainsi dans chacun de ces départements, il a été choisi un site pour cette étude suite à des prospections effectuées dans la région. Le choix des sites a été fait suivant un échantillonnage raisonné. En effet, sachant que les usages faits sur le palmier à huile sont plus ou moins liés aux aspects cultuels, il a été choisi après une prospection un village à majorité chrétienne et animiste, un village à totalité 
musulmane et un village composé de musulmans, de chrétiens et d'animistes. Il s'agit respectivement du village de Carounate dans le département de Oussouye, du village de Kabiline dans le département de Bignona et du village de Kaguite dans le département de Ziguinchor.

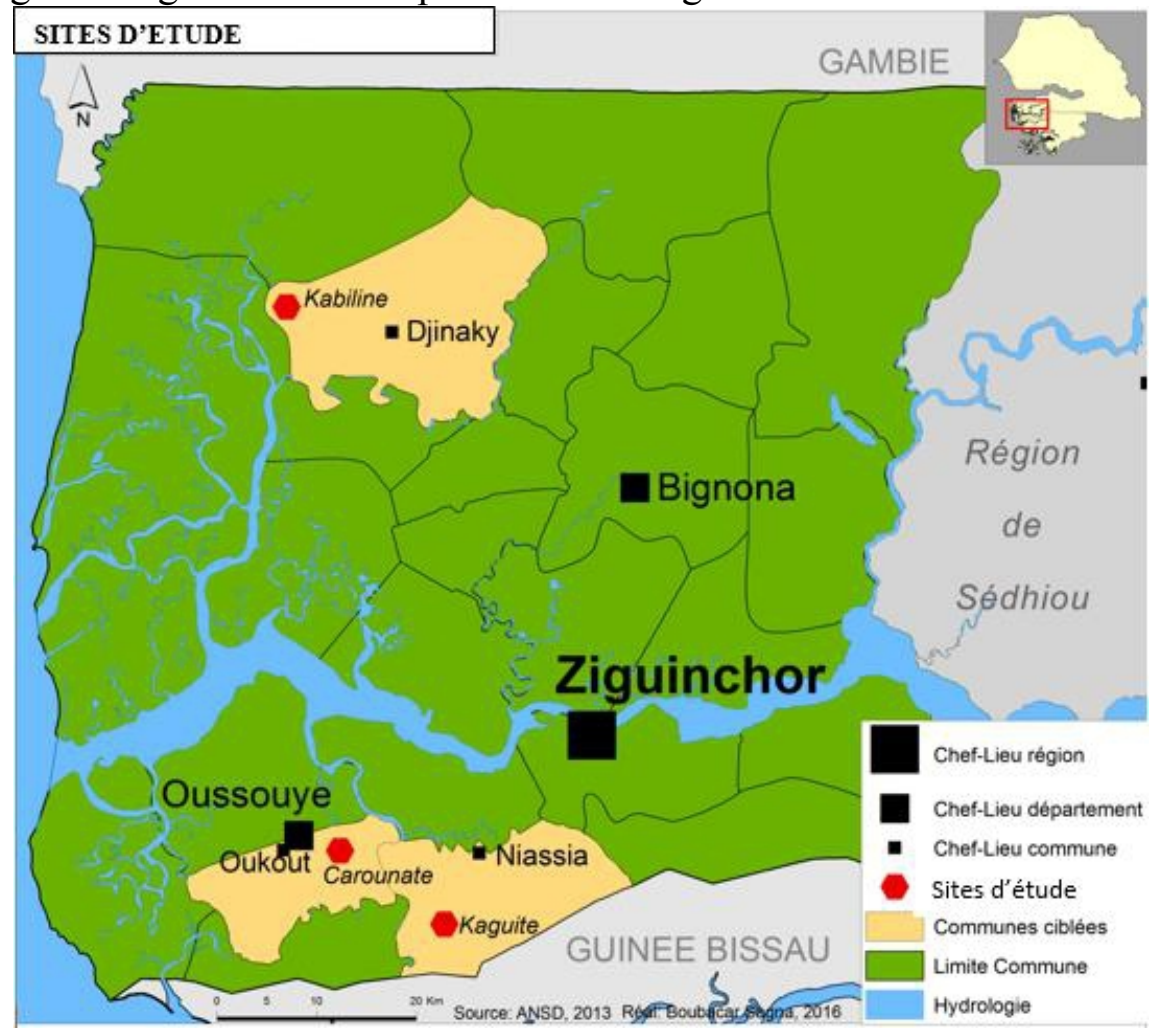

Figure 1: Carte de localisation des sites de l'étude

\section{Enquêtes et entretiens}

Pour faire la caractérisation socioéconomique de Elaeis guineensis en Basse Casamance, des enquêtes ont été menées dans les différents villages. Pour cela, un questionnaire individuel a été administré dans chaque village à un échantillon de la population selon leur secteur d'activité dans la palmeraie. Un taux de sondage de $5 \%$ a été retenu pour la détermination de la taille de l'échantillon. Le nombre d'enquêtés a été calculé à partir de la formule suivante:

\section{Nombre d'enquêtés $=$ Population totale $*$ Taux de sondage}

C'est ainsi que dans le village de Carounate, 33 individus dont 13 femmes ont été enquêtés, 57 individus dont 23 femmes à Kabiline et 56 individus dont 20 femme à Kaguite; soit un total de 146 individus dont 38,36\% de femmes. L’âge minimal des enquêtes est fixé à 18 ans.

A côté du questionnaire, des guides d'entretien ont été administrés aux personnes ressources des villages telles que les chefs de village, deux 
chefs quartier pour chacun des villages de Carounate et de Kaguite et six chefs de quartier de Kabiline. Pour les dirigeants de groupement huit ont été intérogés soit deux par groupement avec deux groupements à Kabiline, un groupement à Carounate et un à Kaguite. Les chefs des services départementaux des Eaux et Forêts ainsi que un des agents ont également été intérogés soit un total de six: deux du département de Oussouye dans compte de Carounate et Kaguite, deux du département de Bignona qui polarise le site de Kabiline.

\section{Traitement des données}

Les données d'enquête ont été d'abord dépouillées manuellement puis saisies et traitées dans le logiciel Sphinx Plus qui permet de générer directement les résultats en fonction des variables de saisie en utilisant les techniques d'analyses uni-variées ou bi-variées. Les premiers résultats ont été transformés sur le tableur Excel pour être présentés sous forme de tableaux, de diagramme et d'histogrammes. Lors du traitement de données d'enquête, la variable la plus utilisée est la Fréquence de Citations (FC) dont la formule de calcul est la suivante :

$$
\mathrm{FC}=\frac{\text { Nombre de citations }}{\text { Nombre total de répondants }} \times 100
$$

Une Analyse en Composantes Principales (ACP) a été également réalisée à l'aide du logiciel XLSTAT.

\section{RESULTATS}

\section{Mode d'exploitation de Elaeis guineensis}

$>$ Mode d'acquisition des produits du palmier à huile

Dans la détermination des modes d'exploitation du palmier à huile, les populations ont été interrogées sur la manière dont elles obtiennent les produits du palmier qu'elles utilisent. Les résultats obtenus sont consignés dans la figure ci-dessous.

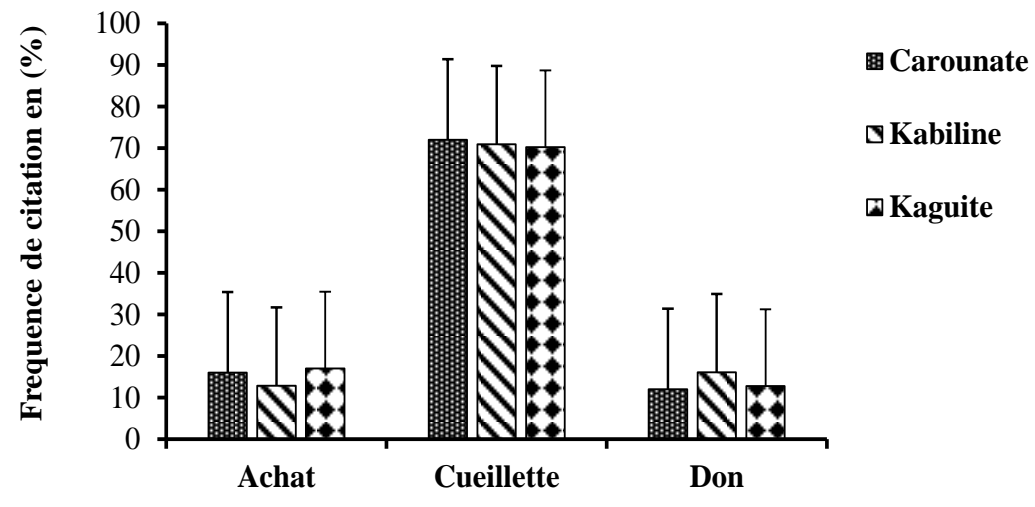

Figure 2 : Mode d'acquisition des noix de palme 
L'analyse de la figure 2 montre que les noix utilisées sont soit achetées, soit cueillies par un membre de la famille ou offertes. Cependant, la majorité de la population enquêtée ( $72 \%$ à Carounate, $70,97 \%$ à Kabiline et 70,21 à Kaguite) obtient leur produit à travers la cueillette. Ce dernier concerne principalement les feuilles que les récolteurs coupent et que les femmes utilisent pour la fabrication des balais.

\section{$>$ Les parties exploitées du palmier à huile et leurs usages}

Les données recueillies à travers les enquêtes de terrain sur les différentes parties du palmier à huile exploitées par la population locale ainsi que les usages qu'elle en fait sont consignées dans le tableau 1.

Tableau 1: Les différentes parties du palmier à huile exploitées et leurs usages

\begin{tabular}{|c|c|c|c|c|c|c|c|}
\hline \multirow{2}{*}{ Partie } & \multicolumn{3}{|c|}{$\begin{array}{l}\text { Fréquence de citation des } \\
\text { parties }\end{array}$} & \multirow{2}{*}{ Usage } & \multicolumn{3}{|c|}{$\begin{array}{l}\text { Fréquence de citation des } \\
\text { usages }\end{array}$} \\
\hline & $\begin{array}{c}\text { Carounat } \\
\mathrm{e}\end{array}$ & $\begin{array}{c}\text { Kabilin } \\
\mathrm{e}\end{array}$ & $\begin{array}{c}\text { Kaguit } \\
\mathrm{e}\end{array}$ & & $\begin{array}{c}\text { Carounat } \\
\mathrm{e}\end{array}$ & $\begin{array}{c}\text { Kabilin } \\
\mathrm{e}\end{array}$ & $\begin{array}{c}\text { Kaguit } \\
\mathrm{e}\end{array}$ \\
\hline \multirow{11}{*}{ Feuille } & \multirow{11}{*}{100} & \multirow{11}{*}{100} & \multirow{11}{*}{100} & Bois de chauffe & 0 & 14,29 & 0 \\
\hline & & & & Meubles & 0 & 7,14 & 0 \\
\hline & & & & Natte & 0 & 7,14 & 0 \\
\hline & & & & Balai & 84,62 & 100 & 94,29 \\
\hline & & & & Clôture & 46,15 & 75 & 77,14 \\
\hline & & & & Plafond & 0 & 96,43 & 60 \\
\hline & & & & Entonnoir & 23,08 & 0 & 8,57 \\
\hline & & & & Fertilisant & 23,08 & 0 & 8,57 \\
\hline & & & & Ceinture & 7,69 & 14,29 & 8,57 \\
\hline & & & & Corde & 7,69 & 17,86 & 8,57 \\
\hline & & & & Palissade & 0 & 7,14 & 0 \\
\hline \multirow{4}{*}{ Fleur } & \multirow{4}{*}{100} & \multirow{4}{*}{100} & \multirow{4}{*}{95,12} & Insectifuge & 46,15 & 0 & 45,71 \\
\hline & & & & Grillade de poisson & 53,85 & 10,71 & 11,43 \\
\hline & & & & Récolte de miel & 0 & 17,86 & 17,14 \\
\hline & & & & Fertilisant & 46,15 & 92,86 & 54,29 \\
\hline \multirow{10}{*}{$\begin{array}{l}\text { Régim } \\
\mathrm{e}\end{array}$} & \multirow{10}{*}{100} & \multirow{10}{*}{100} & \multirow{10}{*}{100} & Huile de palme & 84,62 & 100 & 100 \\
\hline & & & & Huile de palmiste & 0 & 100 & 51,43 \\
\hline & & & & Soupe (à base du jus) & 38,46 & 53,57 & 62,86 \\
\hline & & & & Alimentation des porcs & 23,08 & 0 & 8,57 \\
\hline & & & & Allumage & 7,69 & 67,86 & 8,57 \\
\hline & & & & Conservation du bois & 7,69 & 0 & 0 \\
\hline & & & & Remblayage & 0 & 0 & 2,86 \\
\hline & & & & Savon & 7,69 & 32,14 & 54,29 \\
\hline & & & & Bois de chauffe & 0 & 7,14 & 0 \\
\hline & & & & «Egnob» (Pulpe+sucre) & 7,69 & 3,57 & 11,43 \\
\hline \multirow{3}{*}{ Sève } & \multirow{3}{*}{100} & \multirow{3}{*}{0} & \multirow{3}{*}{41,46} & Consommation & 76,92 & 0 & 31,43 \\
\hline & & & & Libation & 46,15 & 0 & 20,00 \\
\hline & & & & Dot & 7,69 & 0 & 2,86 \\
\hline \multirow{3}{*}{ Stipe } & \multirow{3}{*}{100} & \multirow{3}{*}{100} & \multirow{3}{*}{100} & Bois de chauffe & 0 & 7,14 & 0 \\
\hline & & & & Construction & 100 & 100 & 100 \\
\hline & & & & Fertilisant & 7,69 & 0 & 2,86 \\
\hline
\end{tabular}




\begin{tabular}{|c|c|c|c|c|c|c|c|}
\hline \multirow{3}{*}{ Racine } & \multirow{3}{*}{30} & \multirow{3}{*}{46,43} & \multirow{3}{*}{56,10} & Pharmacopée & 7,69 & 39,29 & 45,71 \\
\hline & & & & Instrument de musique & 7,69 & 0,00 & 5,71 \\
\hline & & & & Fertilisant & 7,69 & 7,14 & 2,86 \\
\hline
\end{tabular}

L'analyse du tableau 1 montre que toutes les parties du palmier à huile sont exploitées par la population locale pour divers usages. Ainsi les usges les plus communément cités sont la construction, la production d'huile de palme et la confection de balais.

\section{* Les feuilles}

Les utilisations faites à partir des feuilles sont très nombreuses et diverses. Les nervures principales ou les feuilles entières sont utilisées dans tous les villages pour la clôture des toilettes traditionnelles et des jardins de case. Les nervures principales servent également à la fabrication des ceintures utilisées pour grimper les palmiers ou les rôniers. Elles sont utilisées comme bois de service pour le plafonnage des maisons dans le village de Kabiline et de Kaguite mais aussi peuvent servir de cordes pour attacher les clôtures ou les fagots de bois. Les feuilles de palmiers brulées ou décomposées sont utilisées comme fertilisant. La nervure des folioles est utilisée pour la confection des balais. La dernière feuille sert à attacher les gerbes de riz.

Dans le village de Kabiline, les feuilles sont utilisées pour la fabrication des palissades, des nattes mais aussi comme bois de chauffe.

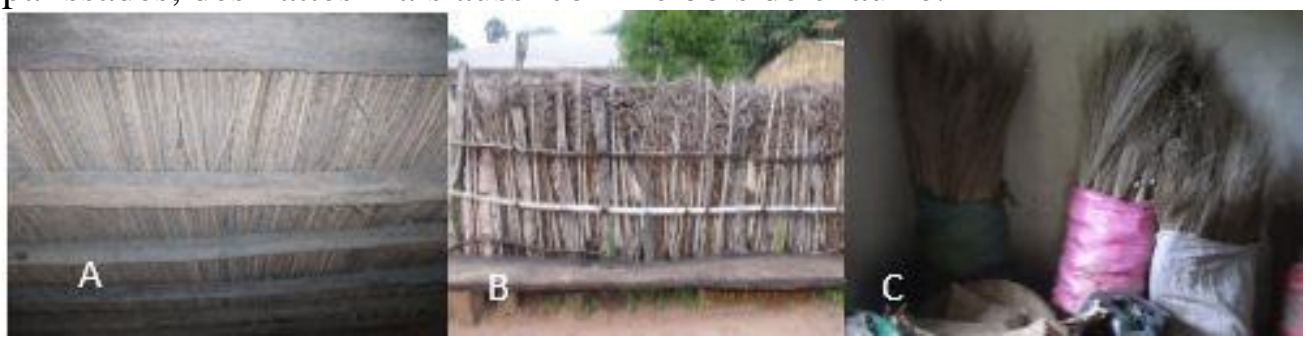

Figure 3:Usages de la feuille du palmier A: Plafond, B: Clôture C: balais

\section{Les fleurs}

Les fleurs du palmier à huile sont utilisées dans l'arboriculture comme fertilisant. En effet, selon la population les fleurs permettent non seulement d'augmenter la fertilité des sols mais aussi de conserver pendant longtemps l'humidité du sol. Elles sont aussi utilisées pour la grillade et le fumage de poisson. Les fleurs sont utilisées comme insectifuges. En effet, la fumée de la fleur sèche allumée est utilisée pour chasser les insectes comme les abeilles pendant la récolte du miel des ruches traditionnelles, mais aussi pour embaumer les animaux domestiques afin de les débarasser des insectes (tiques et acariens.....). 


\section{* Les régimes}

Les produits provenant du régime comme la pulpe, l'huile de palme, la soupe et l'huile de palmiste, sont beaucoup utilisés dans l'alimentation des populations locales. La production de l'huile de palmiste n'est pas notée à Carounate du fait que les noix sont utilisées pour l'alimentation des porcs. Les résidus de la pulpe, constitués de fibres, après extraction de l'huile de palme sont utilisés à l'état sec pour allumer le feu surtout pendant la saison des pluies. L'huile de palmiste est aussi utilisée dans la fabrication du savon traditionnel après fermentation.

Les noix sont utilisées à la place du bois de chauffe à Kaguite dans la production de l'huile de palme et après concassage de noix pour la production de l'huile de palmiste, les coques sont utilisées pour remblayer les toilettes. A Kabiline, les grappes sèches sont utilisées comme bois de chauffe lors de la production de l'huile de palme.

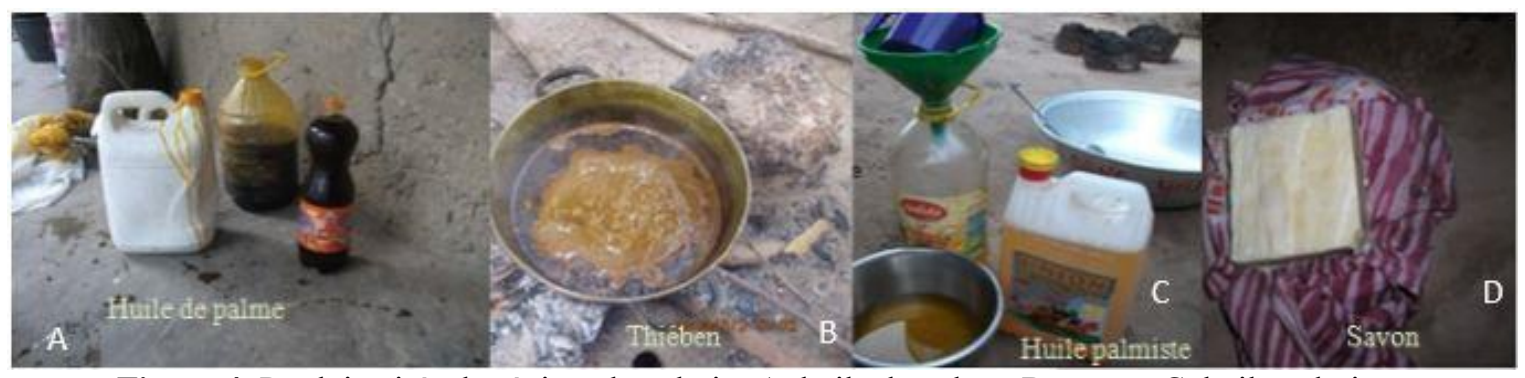

Figure 4: Produits tirés du régime de palmier A: huile de palme, B: soupe, C: huile palmiste, D: savon

\section{* La sève}

Elle est exploitée uniquement dans les villages de Carounate et de Kaguite où on retrouve des chrétiens et animistes. Le vin, sève fermentée, est beaucoup utilisé comme boisson par la population locale. Il est aussi utilisé dans beaucoup de cérémonies traditionnelles comme les mariages mais aussi pour les libations. Le vin de palme n'est pas exploité ni consommé à Kabiline où la population est constituée uniquement de musulmans.

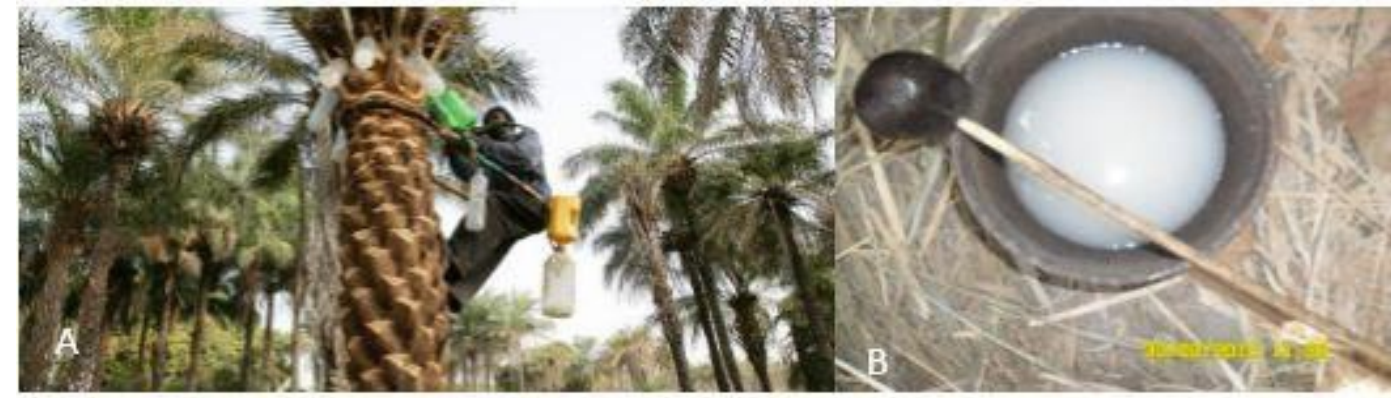

Figure 5: A : Récolteur de vin, B : vin de palme non fermenté 


\section{Le stipe}

Le stipe est beaucoup utilisé dans la construction pour la confection des charpentes et des toits. La poudre issue de la décomposition du stipe est utilisée comme fertilisant dans le village de Carounate et de Kaguite. A Kabiline, les stipes de palmiers morts sont parfois utilisés comme bois de chauffe et pour la confection de ruches traditionnelles.

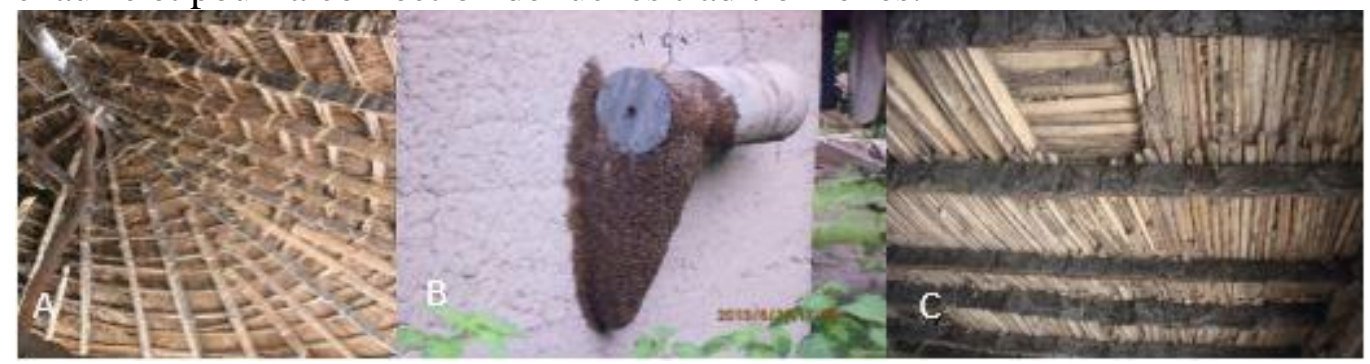

Figure 6:Usages du stipe du palmier A: Charpente, B: Ruche traditionnelle C : Plafond

* Les racines

Elles sont utilisées dans la pharmacopée dans tous les villages par la population locale. En effet les racines du palmier à huile sont utilisées pour soigner les maux de dents à partir de la vapeur venant de leur décoction. L'eau tirée de cette décoction, prise comme boisson, permet de soigner la syphilis et les maux de ventre des femmes après accouchement. Elles servent également à soigner l'impuissance sexuelle, l'obésité, mais aussi de renforcer la résistance physique des nouveaux nés.

Auparavant, à Carounate et à Kaguite, la fibre interne de la racine servait à la fabrication des guitares traditionnelles appelées «ékonting» en diola.

A Kabiline et à Kaguite, les racines de palmiers morts sont utilisées comme fertilisants.

$>$ Contribution du palmier à huile à l'alimentation des populations Afin d'évaluer la place qu'occupent les produits du palmier à huile dans l'alimentation de la population ainsi que la dynamique de la consommation de ces produits, des enquêtes ont été menées auprès de cette dernière. Les résultats trouvés sont résumés dans la figure 7. 


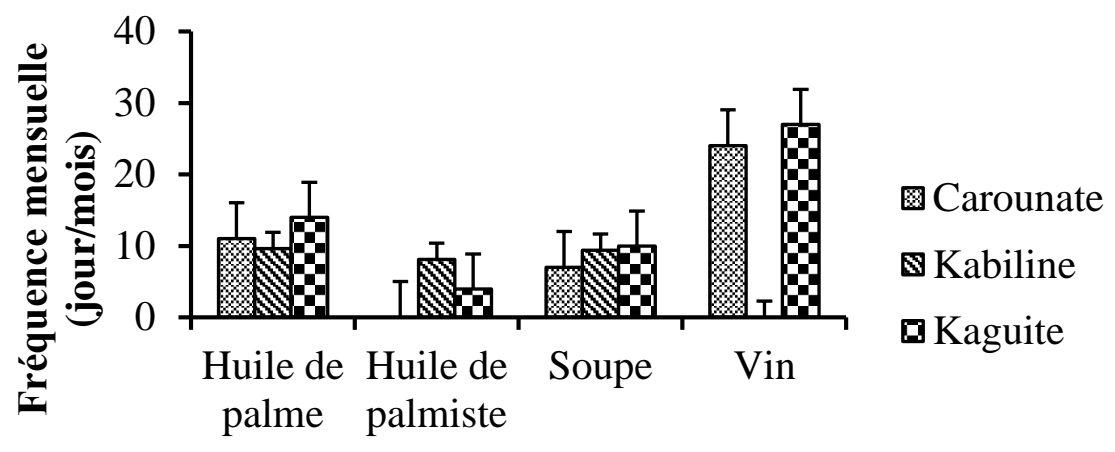

\section{Produit consommé}

Figure 7: Fréquence mensuelle de consommation des produits du palmier à huile

L'analyse de la figure 7 montre que l'huile de palme, l'huile de palmiste, la soupe et le vin sont les produits du palmier qui sont fréquament consommés. Cependant, la fréquence de consommation varie d'une famille à une autre et d'une localité à une autre. En effet, le vin de palme est le produit le plus consommé à Carounate et à Kaguite avec une fréquence moyenne respective de 24 et 27 jours par mois. En dehors du vin, l'huile de palme est plus consommée dans tous les villages. Elle est suivie de la soupe et de l'huile de palmiste. Cette dernière n'est pas consommée à Carounate car la population, constituée de chrétiens et animistes, utilise souvent les noix pour l'alimentation des porcs. Elle est peu consommée à Kaguite parce que le village ne dispose pas de machine pour concasser les noix.

Il est nécessaire de signaler que les fréquences moyennes de consommation représentées dans la figure 7 ne sont pas fixes, elles dépendent de la disponibilité des produits. L'évolution de la fréquence de consommation de ce produit est consignée dans la figure 8.

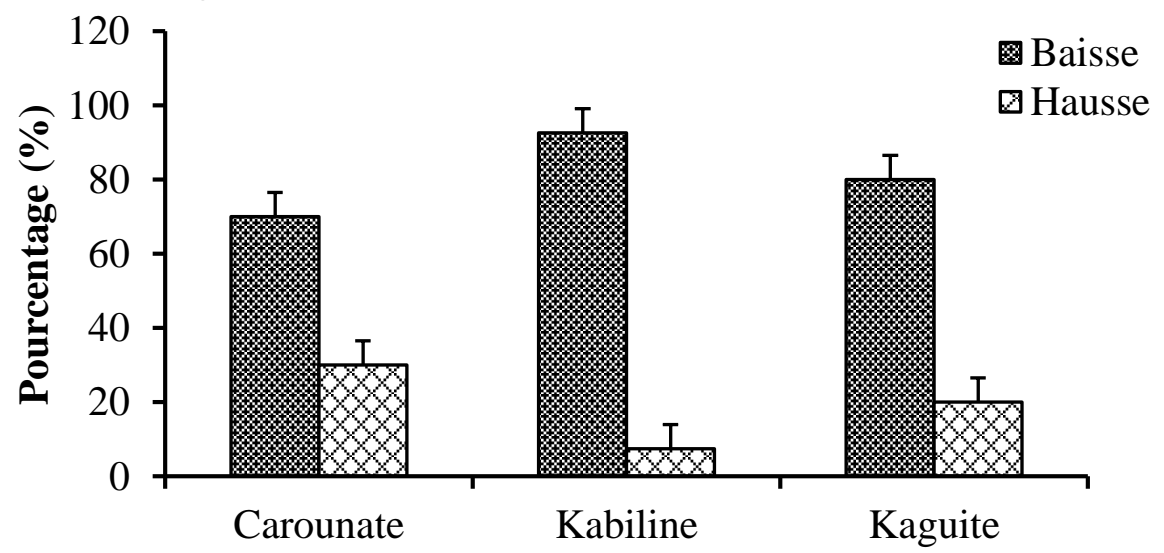

Figure 8: Dynamique de consommation des produits du palmier à huile

Le constat général dans les trois sites est une évolution régressive de la consommation des produits tirés du palmier à huile comparé aux temps passés. En 
effet, $70 \%$ des enquêtés à Carounate, $92,59 \%$ à Kabiline et $80 \%$ à Kaguite affirment que la consommation des produits du palmier, principalement celle de l'huile de palme, a baissé. Cette baisse de la consommation s'explique par la cherté des produits et la forte demande. La grande partie de l'huile de palme produite est acheminée vers les grands marchés, particulièrement ceux de Dakar mais parfois de Ziguinchor causant un manque de ce produit à un certain moment de l'année. La hausse de la consommation concerne principalement le vin car certains des enquêtés de Carounate et de Kaguite disent que les jeunes boivent maintenant plus qu'avant. Les $30 \%$ des enquêtés de Carounate, $7,41 \%$ de Kabiline et $20 \%$ de Kaguite qui parlent d'augmentation de la consommation se basent sur le fait qu'il y a un exploitant chez eux.

$>$ Contribution du palmier à la génération de revenus pour les populations Afin d'évaluer l'apport du palmier à huile au revenu des populations locales, des questions ont été posées à ces dernières lors des enquêtes afin de déterminer les produits vendus mais aussi d'estimer la valeur économique de chacun. Les résultats obtenus sont condensés dans le tableau ci-dessous.

Tableau 2: Les produits du palmier vendus et leur valeur économique

\begin{tabular}{|c|c|c|c|}
\hline Village & Produits & $\begin{array}{c}\text { Fréquence de } \\
\text { citation }\end{array}$ & $\begin{array}{c}\text { Revenu annuel d'une Famille } \\
\text { exploitante (f CFA) }\end{array}$ \\
\hline \multirow{4}{*}{ Carounate } & Huile de palme & 66,67 & De 26000 à 135000 \\
\cline { 2 - 4 } & Huile de palmiste & 0 & 0 \\
\cline { 2 - 4 } & Vin & 61,11 & De 180000 à 1800000 \\
\cline { 2 - 4 } Kabiline & Balais & 66,67 & De 2500 à 60000 \\
\hline \multirow{4}{*}{ Kaguite } & Huile de palme & 75 & De 10000 à 200000 \\
\cline { 2 - 4 } & Huile de palmiste & 46,43 & De 10000 à 120000 \\
\cline { 2 - 4 } & Vin & 0 & 0 \\
\cline { 2 - 4 } & Balais & 50 & De 5000 à 30000 \\
\cline { 2 - 4 } & Huile de palme palmiste & 100 & De 10000 à 1350000 \\
\cline { 2 - 4 } & Vin & 20 & De 10000 à 15000 \\
\cline { 2 - 4 } & Balais & 70 & De 375000 à 675000 \\
\hline
\end{tabular}

L'analyse du tableau 2 montre que les produits vendus du palmier à huile tels que l'huile de palme, l'huile de palmiste, le vin et les balais contribuent fortement à l'augmentation des revenus des populations locales. Parmi ces produits, c'est le vin qui a une plus grande valeur économique. En effet, à Carounate et Kaguite, un récolteur peut gagner en moyenne respectivement $739125 \mathrm{f}$ et $537500 \mathrm{f}$ CFA par an. Cependant le vin n'est pas récolté dans le village de Kabiline. Apres le vin de palme, vient l'huile de palme qui fournit annuellement un revenu important aux producteurs (46375f à Carounate, $81452 \mathrm{f}$ à Kabiline et $218533 \mathrm{f}$ à Kaguite). Dans le village de Kabiline, la vente de l'huile de palme uniquement peut faire entrer 
dans les caisses du village une somme globale moyenne de 3463700 f CFA par an, soit 865925f CFA par quartier. La particularité de Kabiline est que l'exploitation ce fait par un groupement et les sommes sont versées dans la caisse du village. C'est aprés l'exlpoitation dans le compte du village qu'il est permis au individuels de récolté dans leur propre compte La vente de balais fournit également aux producteurs un revenu annuel non négligeable. L'huile de palmiste n'est pas vendue à Carounate et peu vendu à Kaguite où les noix sont utilisées pour l'alimentation des porcs. Le bois n'est vendu dans aucun des villages, il n'est coupé qu'en cas de besoin pour la construction de maison.

Les variables telles que la fréquence de consommation, la dynamique de consommation, le revenu moyen fourni par les produits ont été regroupées pour faire une ACP. Les résultats obtenus sont consignés dans la figure 9. Avec l'axe F1 représentant les consommations de l'huile palmiste, du vin de palme, la fluctuation de la consommation (baisse et hausse) et les revenus tirés de la vente l'huile palmiste et du vin de palme. L'axe F1 correspond l'huile palmiste, la consommation, les revenus tirés de la vente l'huile de palme et du balais.

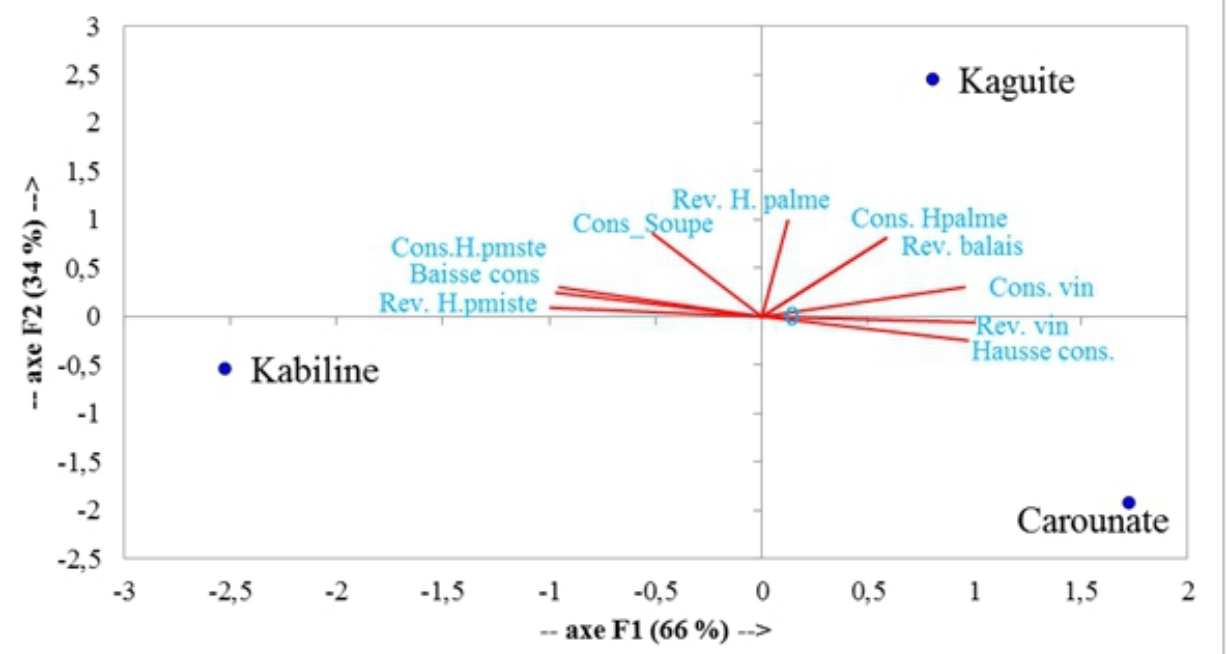

Figure 9: Répartition des sites en fonction des paramètres socioéconomiques

L'analyse de la figure 9 montre que l'huile de palmiste et la soupe sont plus consommées dans le village de Kabiline. L'huile de palmiste y est également plus vendue. Cependant, la fréquence de consommation de ces produits a généralement baissé comparé au temps passé. Dans le village de Kaguite, c'est l'huile de palme et le vin qui sont les plus consommés. Le revenu moyen y est aussi important pour ce qui concerne l'huile de palme et les balais. A Carounate, le vin donne aux exploitants un revenu très important et la consommation en produits du palmier à huile a généralement augmenté. 


\section{$>$ Autres activités menées dans la palmeraie}

En dehors des différents produits tirés du palmier à huile, un certain nombre d'activité est réalisée dans la palmeraie. La détermination de ces activités a été faite à travers le questionnaire administré aux populations. Les résultats sont consignés dans la figure 10 .

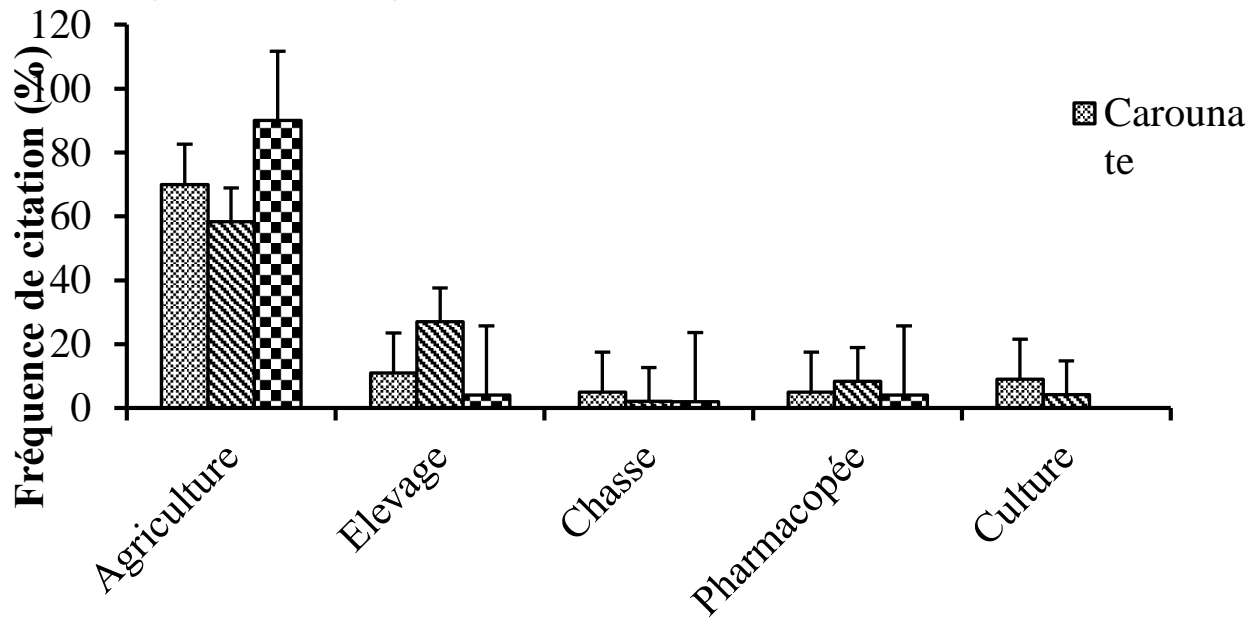

Figure 10: Autres activités réalisées dans la palmeraie

L'analyse de la figure 10 montre que l'agriculture, le pâturage, la pharmacopée, les activités culturelles et la chasse sont les activités généralement menées dans la palmeraie. Cependant parmi ces activités, l'agriculture domine largement. En effet, $70 \%$ des enquêtés à Carounate, $58,33 \%$ à Kabiline et $90 \%$ à Kaguite affirment qu'ils pratiquent l'agriculture dans la palmeraie. Les spéculations les plus cultivées dans les parcs ainsi que la nature de l'association sont consignées dans la figure 11 .

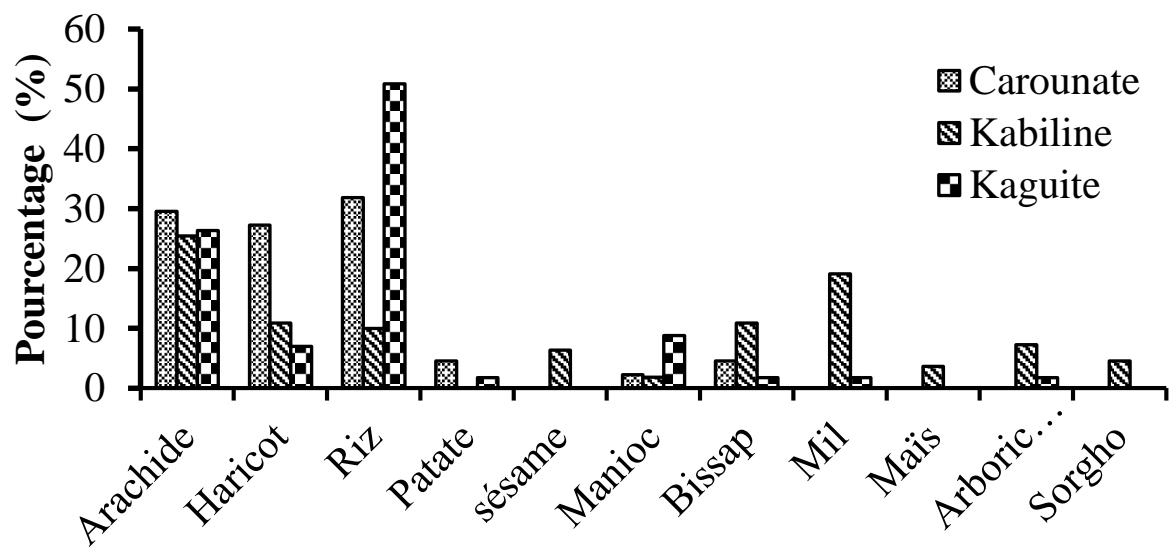

Figure 11: Cultures associées au palmier à huile 
Différentes spéculations sont cultivées sous les palmiers. Cependant les plus fréquentes dans tous les villages sont l'arachide, le riz et l'arachide. Dans le village de Kabiline, le mil est aussi beaucoup cultivé dans la palmeraie.

Concernant la nature de l'interaction entre les palmiers et les cultures, $100 \%$ des enquêtés de Carounate et de Kabiline et 96,55\%(tableau 3) de Kaguite affirment qu'il y a une interaction positive. Les 3,45\% de Kaguite restant pensent qu'il n'y a pas d'effet significatif.

Tableau 3: Interaction palmier/cultures et Importance socioéconomique du palmier

\begin{tabular}{crr}
\hline & \multicolumn{1}{c}{ Interaction positive } & Importance du palmier \\
\hline Kabiline & $100 \%$ & $37,41 \%$ \\
\hline Carounate & $100 \%$ & $90,91 \%$ \\
\hline Kaguite & $96,50 \%$ & $45,71 \%$ \\
\hline
\end{tabular}

\section{$>\quad$ Importance socioéconomique du palmier à huile}

Dans l'ensemble de la zone, la population affirme que les produits issus $\mathrm{du}$ palmier à huile occupent une place très importante dans leur vie et contribuent fortement à leur bien-être (tableau 3). C'est ainsi que $(90,91 \%$ à Carounate, $37,41 \%$ à Kabiline et $45,71 \%$ à Kagite) affirment que l'exploitation du palmier à huile occupe la deuxième place, après l'agriculture, dans leurs activités. En effet, d'après les enquêtés, c'est avec les revenus tirés de la palmeraie qu'ils assurent l'alimentation de la famille, la santé, la scolarité, l'habillement, etc. de leurs enfants.

Dans le village de Kabiline, c'est avec les revenus provenant de la vente de l'huile de palme, que les populations organisent les événements religieux et leurs congrès annuels. Cet argent est aussi utilisé pour la construction de leur école et leur mosquée. Il est également utilisé dans le domaine sportif pour la prise en charge de leur équipe de championnat populaire et pour chaque cotisation du village.

\section{DISCUSSION}

La presque totalité des parties du palmier à huile est exploitée par les populations locales pour divers usages. Ces usages peuvent être classés dans différents domaines socio-économique et écologique.

Pour ce qui est du bien-être social, le stipe est utilisé dans la construction pour la confection des charpentes et des toits. Les nervures principales des feuilles sont également utilisées dans la construction pour la confection des plafonds. Ces résultats corroborent ceux d'autres auteurs tels que Arbonnier et al., (2008), Carrere (2010) et Sagna 2016 selon qui, le palmier à huile fournit des matériaux de construction. Les usages comme les clôtures, balais, cordes bois de chauffe etc. contribuent également au bien-être social de la population. 
Sur le plan économique, la vente des produits du palmier à huile tels que le vin de palme, les huiles et les balais donne des revenus importants aux producteurs et exploitants et contribue beaucoup à l'amélioration de leur situation financière. Ce résultat est en accord avec ceux de Ofosu-Budu et Sarpong (2013), Gomis (2015) et Sagna (2016) qui affirment que les palmeraies et les huileries, ainsi que l'ensemble des services liés à la production, ont contribué et continuent de nos jours à contribuer au développement des économies locales en Guinée Forestière et en Basse Guinée. Ce qui fait du palmier à huile une source d'économie traditionnelle (Yombouno, 2014).

Dans le domaine de l'alimentation, les produits comme l'huile de palme, la soupe, «égnob » et l'huile de palmiste occupent une place très importante dans l'alimentation de la population locale. Ce résultat corrobore ceux de plusieurs auteurs. C'est ainsi que Angerand (2007), Gomis (2015) et Sagna 2016 affirment que ces huiles sont à la base de nombreux régimes alimentaires, notamment en Afrique. Selon Goudiaby (2013), les parcs agroforestiers contribuent fortement à la subsistance de la population locale. En plus de l'alimentation, l'huile de palme est également utilisée dans la fabrication de savon. Ce résultat est en accord avec Ofosu-Budu et Sarpong (2013) selon qui, le palmier à huile, un oléagineux essentiel, fournit beaucoup de produits, à la fois pour la consommation domestique et comme matière première pour le secteur de l'industrie. Le vin de palme est aussi beaucoup consommé dans les villages de Carounate et de Kaguite.

Dans le domaine de la santé, le palmier à huile est beaucoup utilisé dans la pharmacopée pour soigner un certain nombre de maladie comme la syphilis, les maux de ventre, les maux de dents, l'impuissance sexuelle, l'obésité etc. Ce résultat est en accord avec celui de certains auteurs comme Arbonnier (2000), Diatta et Sagna (2013) et Arbonnier et al., (2008) selon qui, le palmier à huile est beaucoup exploité pour la médecine traditionnelle.

Dans le domaine culturel et cultuel, le vin de palme, boisson alcoolisée naturelle extraite du palmier est incontournable lors des libations et l'organisation de certaines cérémonies comme les mariages dans les villages de Carounate et Kaguite pour la communauté chrétienne et animiste. Selon Ofosu-Budu et Sarpong (2013), cette boisson traditionnelle héritée des ancêtres est très souvent utilisée lors des cérémonies de réjouissance comme les mariages, les rites coutumiers ou religieux etc. Selon Yombouno, (2014), le palmier à huile comporte des valeurs socioculturelles importantes.

Dans le domaine écologique, les enquêtes réalisées dans la Basse Casamance ont montré que, parmi les diverses activités qui sont menées dans la palmeraie, l'agriculture domine largement. La majorité des enquêtés ont affirmé que l'interaction palmier-culture est positive. En effet selon eux, le palmier à huile augmente la fertilité des sols et son effet d'ombrage n'est pas 
significatif s'il est élagué. Ce résultat est en accord avec celui de Akouehou et al., (2013) qui affirme que dans le département de l'Atlantique (Sud du Bénin), les raisons qui ont motivé les producteurs à maintenir une densité fortes de palmier à huile avec des cultures annuelles sont liées à sa capacité à remonter la fertilité des terres.

\section{Conclusion}

Elaeis guineensis occupe une place prépondérante dans la subsistance des populations de la Basse Casamance. C'est pourquoi il est l'une des espèces forestières les plus exploitées pour ses nombreux services et produits. Cependant, cette espèce est sujette à de réelles menaces liées à une combinaison de facteurs naturels et anthropiques. Cette étude qui s'était fixé comme objectif une évaluation des biens et services fournis par le palmier à huile en Basse Casamance a permis de montrer que celui-ci contribue fortement au bien-être de la population particulièrement dans l'alimentation et l'économie.

Au vu de son importance il est impératif d'assurer la pérennisation de ces systèmes de production à travers :

* La valorisation et l'exploitation durable des palmeraies naturelles en Basse Casamance en prenant soin des régénérations;

* Le renouvellement des palmeraies vieillissantes au risque de voir ces systèmes séculaires disparaitre;

- La promotion, après une étude approfondie, de l'introduction de variété améliorée (comme tenera) pour diversifier la production et augmenter la disponibilité d'huile de palme en Basse Casamance tout en conservant les palmiers locaux;

* La mécanisation de la production d'huile de palme et d'huile de palmiste afin d'optimiser les rendements;

La mise en place un cadre de concertation multi-acteurs autour de la gestion de la palmeraie.

\section{References :}

1. Akouehou G. S., Assogba D. O., Houndonougbo A. et Sinsin A. B., 2013. -Diversité floristique, sécurisation foncière et gestion des systèmes agroforestiers à palmier à huile (Elaeis guineensis) en zones périurbaines et rurales du Département de l'Atlantique au Sud du Bénin. ISSN 1991-8631. 10 pages.

2. Angerand S. 2007- FIPCAM Hold up dans les forêts Camerounaise: Les Amis de la terre 13 pages.

3. Arbonnier M. 2000. Arbres, arbustes et lianes des zones sèches d'Afrique de l'Ouest. Montpellier : CIRAD, MNHN, $541 \mathrm{p}$ 
4. Bonnet P., Arbonnier M., Grard P., 2008. Versailles : Ed. Quae, 1 Cd-Rom.

5. Carrere, R., 2010; -Le palmier à huile en Afrique : le passé, le présent et le futur. Mouvement mondial pour les Forêts tropicales. Collection du WRM sur les plantations $\mathrm{N}^{\circ} 15$.

6. Diatta E. A. et. Sagna B., 2013.-Caractérisation et modes d'exploitation des parcs à Elaeis guineensis, Jacq. en Basse Casamance : cas des villages de Carounate (cassa) et de Djiguinoume (kalounayes). Mémoire de Licence Agroforesterie, Université Assane de Ziguinchor. 49 pages.

7. Ezebilo, E.E. and Mattsson, L. 2010. Socio-economic benefits of protected ereas as percived by local people arround cross river National Park, Nageria Forest Policy and Economic, 12 ; 189-193.

8. Gomis Z. D., 2015. -Les parcs agroforestiers à Elaeis guineensis Jacq. (Palmier à huile) : Caractéristiques biophysiques et importance socio-économique à Ouonck (Basse-Casamance). Mémoire de Master 2. Université Assane de Ziguinchor. 79 pages.

9. Goudiaby M., 2013. Les parcs agroforestiers en Basse-Casamance : Contribution du Parkia biglobosa (néré) à la réduction des risques de pauvreté des ménages de la communauté rurale de Mangagoulack, au Sénégal. Université de Laval, Québec, Canada : 98 pages.

10. Niane A., 1984. -Etudes cartographiques et agro-pédologiques des sols de plateau de basse Casamance. Mémoire de Stage, ISRA. 104 pages.

11. Ofosu-Budu, K., et Sarpong D., 2013.-Croissance de la filière de l'huile de palme au Ghana : implications pour les petits agriculteurs et viabilité à long terme, Dans : Reconstruire le potentiel alimentaire de l'Afrique de l'Ouest, A. Elbehri (ed.), FAO/FIDA.

12. Sagna B., 2016- Paramètres structuraux, modes de gestion et importance socio-économique des parcs agroforestiers à Elaeis guineensis Jacq. (Palmier à huile) en Basse-Casamance. Mémoire de master Université Assane SECK de Ziguinchor 55 pages.

13. Yombouno. A. -2014.-Historique de l'exploitation du palmier à huile en Afrique. 36 pages. 